\title{
The lymphatic vasculature revisited
}

\author{
Dontscho Kerjaschki
}

Clinical Department of Pathology, Medical University of Vienna, Vienna, Austria.

\begin{abstract}
Lymphatic vessels constitute a ubiquitous countercurrent system to the blood vasculature that returns interstitial fluid, salts, small molecules, resorbed fat, and cells to the bloodstream. They serve as conduits to lymph nodes and are essential for multiple physiologic activities. However, they are also hijacked by cancer cells to establish initial lymph node metastases, as well as by infectious agents and parasites. Despite these obvious important functions in human pathologies, a more detailed understanding of the molecular mechanisms involved in the regulation of the lymphatic vasculature has trailed that of the blood vasculature for many years, mainly because critical specific characteristics of lymphatic endothelial cells were discovered only recently. In this Review series, several major aspects of the active and passive involvement of the lymphatic vasculature in human disease and physiology are presented, with a focus on translational findings.
\end{abstract}

Many scientific fields develop in waves that vary in frequency and amplitude, with rapid progress initiated by serendipitous findings in seemingly unrelated fields. Research on the structure and function of the lymphatic vasculature exemplifies this model. As presented in this Review series, novel insights into the involvement of lymphatic vessels in human disease and physiology have not only provided additional information on pathogenesis, but have changed the prevailing paradigms of disease mechanisms as a prelude to improved targeted therapies.

Historically, the identification of lymphatic vessels relied on their content, known as "white blood" (1). Their macroscopic visualization was made possible by intradermal injection of colloid tracers in fresh cadavers, and striking examples of the results are provided by the anatomical wax models produced in Florence around 1770 and on display in the Josephinum in Vienna (www. josephinum.ac.at). A model of the cervical lymphatic network is depicted on the cover of this series. These techniques primarily detect large branches of the lymphatic system, i.e., the collecting vessels that serve as peristaltic pumps to move the lymphatic fluid toward the thoracic duct by a precisely coordinated contraction of the smooth muscles in their media, while strategically positioned valves prevent reflux. Recent advances in our understanding of smooth muscle contraction (2) and formation and stability of the valves (3) have clarified these processes. In this series, Eva SevickMuraca and colleagues (4) summarize the current state of in vivo visualization that reveals the lymphatic vasculature in much better resolution. Though our understanding of the collecting vessels has improved, we are progressively gaining detailed knowledge concerning the initial microvascular segment, which is arguably the most functionally relevant part of the vascular lymphatic tree and hence the topic of most Reviews in this series. Under the microscope the initial lymphatic capillaries are composed of sacs of endothelial cells and are devoid of pericytes. These capillaries merge into a precollector vessel segment with few pericytes that opens into the collectors (Figure 1).

At the center of the current increase in lymphatic research are new insights into the biology of the lymphatic endothelial cells (LECs). This phase was heralded by detailed ultrastructural investigations of initial lymphatic vessels (5). The critical breakthrough to a functional understanding was achieved with the discovery of

Conflict of interest: The author has declared that no conflict of interest exists. Citation for this article: J Clin Invest. 2014;124(3):874-877. doi:10.1172/JCI74854. the main lymphangiogenic growth factor, VEGFC, and its receptor, VEGFR3 (6). This was followed by the identification of proteins that discriminate between endothelial cells of the lymphatic and blood vessel lineages (7) that can serve as selective markers for the localization and isolation of LECs $(8,9)$, a development reviewed by Kari Alitalo and colleagues in this series (10).

Since the lymphatic system is as branched and widely distributed as the blood vasculature, it is not surprising that it is involved in most disease processes, ranging from inflammation to metastatic spreading of cancers. It is becoming increasingly clear that the lymphatics do not just serve as passive conduits for interstitial fluid and cells, but are actively involved in disease, as reviewed in detail in this series. Below, I highlight a few selected aspects of current research that could become clinically relevant in the coming years.

\section{Variability of microvascular LECs}

Two populations of LECs that differ in their content of the membrane mucoprotein podoplanin were identified in the dermal microvasculature $(11,12)$ : endothelial cells expressing high levels of podoplanin are localized to the initial capillaries, while those with lower levels of podoplanin are found in precollector segments. Interestingly, these two populations are different lineages that differ in gene expression. This is presumably of functional importance, as the podoplanin-rich cells produce CCL21 that would attract, among others, dendritic cells, while the podoplanin-low cells in the precollectors secrete CCL27, which attracts CCR10+ inflammatory and neoplastic $\mathrm{T}$ lymphocytes. These two lymphatic endothelial sublineages also express different levels of Notch, which regulates podoplanin expression (13). These results provide the first indication for potential functional specialization of LECs in different parts of the lymphatic vasculature that could potentially be exploited to re-route inflammatory and immune cells.

\section{The role of macrophages in lymphangiogenesis}

The perplexing plasticity of monocytes/macrophages has been extended to their involvement in neolymphangiogenesis. They contribute in at least two ways, namely as a source of VEGFC after appropriate stimulation, or by transdifferentiation into LECs that integrate into the growing capillaries (ref. 14 and Figure 2). This was originally shown for tumor-associated macrophages (15) and in kidney transplants in the course of organ rejection (16). Most impressively, transdifferentiation was demonstrated by direct observation in the inflammatory model of corneal wounding, in 


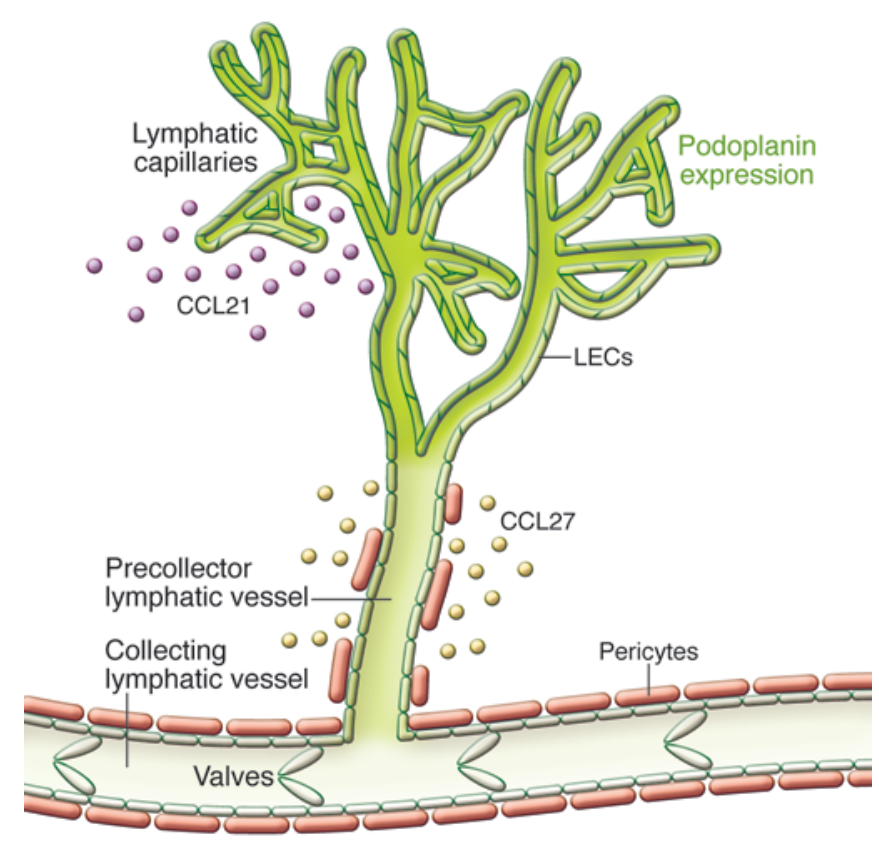

which lymphangiogenesis was abolished when macrophages were depleted (17). As potential lymphangioblastic precursors, a large pool of about $50 \%$ of $\mathrm{CD} 14^{+}$monocytes was identified in the circulation. These monocytes were found to constitutively express VEGFR3 and turn on expression of podoplanin and several other lymphatic markers when cultured for a prolonged period (16). Recent evidence indicates that cells of the adaptive immune system are able to increase (18) or suppress (19) lymphangiogenesis, as reviewed by Gou Young Koh and colleagues (20) in this series. These findings are of potential practical importance, as they could provide the basis for novel therapeutic strategies to support precisely targeted neolymphangiogenesis. An in-depth understanding of the development of the lymphatic vasculature, as presented here by Ying Yang and Guillermo Oliver (21), might also provide further clues on how to regrow lymphatic vessels after interruptions. A practical example is the treatment of secondary lymphedemas that still are a threat after axillary lymphadenoectomy in breast cancer surgery, as detailed in this series by Peter Mortimer and Stanley Rockson (22).

\section{Lymphatics in hypertension and hypercholesterolemia}

A major development of clinical relevance was initiated by the finding that lymphangiogenesis is causally involved in the regulation of $\mathrm{Na}^{+}$storage in the skin and that $\mathrm{Na}^{+}$overload is associated with massive lymphatic neoangiogenesis (23). In rodent models of $\mathrm{Na}^{+}$-induced hypertension, macrophages are attracted to the dermis and start to produce VEGFC. This is driven by the macrophage transcription factor TonEBP, which serves as a specific membrane "osmometer" for $\mathrm{Na}^{+}$hyperosmolality and binds to the promoter of the VEGFC gene in macrophages. Depletion of macrophages or blocking of lymphangiogenesis by specific trapping of VEGFC by a soluble VEGFR3 ectodomain fusion protein resulted in massively increased sensitivity to dietary $\mathrm{Na}^{+}$overload and severe hypertension (24). These results have been extended to humans, as hypertensive patients exhibit high levels of $\mathrm{Na}^{+}$in the skin by NMR and high levels of VEGFC in the circulation (25). This somewhat unex-

\section{Figure 1}

Basic design of the initial segment of the lymphatic microvasculature of the dermis. The initial capillaries start as blind sacs. Their LECs form overlapping junctions, express large amounts of the membrane mucoprotein podoplanin (green), and release the chemokine CCL21. This attracts CCR7+ immune cells, such as dendritic cells and Tregs. The precollectors contain LECs with low amounts of podoplanin, and the LECs produce CCL27, which attracts inflammatory CCR10+ $\mathrm{T}$ lymphocytes. This precollector segment opens into the collecting vessels that are endowed with podoplanin-low endothelial cells and that form valves. Pericytes/mural cells partially cover the precollectors and completely ensheath collecting vessels.

pected finding promises to change the paradigm of the pathogenesis of hypertension and may provide novel therapeutic targets for this common disease.

In this series, Gwendalyn Randolph and Norman Miller (26) present another groundbreaking concept that assigns the lymphatic microvasculature a key role in the transport of cholesterol-rich lipoproteins from the interstitium to the circulation. Predictably, these results will be of relevance for controlling hypercholesterolemia.

\section{Lymphangiogenesis in transplant rejection}

A previously unknown and poorly understood phenomenon is the massive growth of lymphatic vessels in the rejection of kidney (27) and heart (28) transplants. The contribution of lymphatic vessels to the reorganization of the inflammatory rejection infiltrates in the formation of tertiary lymphatic organs particularly requires clarification, as clinicopathological studies have documented the counterintuitive positive correlation between lymphatic vessel density and clinical outcome in human renal transplants (29). In this series, Nancy Ruddle (30) and Melody Swartz and colleagues (31) discuss the roles of the lymphatic system in the formation of tertiary lymphatic organs and more generally in the development of the adaptive immune response, respectively. Understanding and exploiting the underlying mechanisms that regulate the traffic of immune cells across lymphatic vascular walls may contribute to the support of graft tolerance and to the course of inflammation and immune responses in general.

\section{Lymph node metastasis}

Carcinomas invariably form the first detectable metastases in regional (sentinel) and subsequently post-regional lymph nodes. It is established that this predicts the formation of distant organ metastases and thus the patient's outcome. Despite the obvious clinical importance of tumor cell invasion into lymphatic vessels and colonization of the lymph nodes, current knowledge relies primarily on correlative studies. Mechanistic insights are only just beginning to emerge and comprise a better understanding of functions of the tumor microenvironment (32), the biochemistry of tumor cell invasion into lymphatics (33), and the transformation of the lymph node sinus into labyrinths that are lined by lymphatic endothelial-like cells (34), as reviewed in this series by Sinem Karaman and Michael Detmar (35). The knowledge in this field is not yet sufficiently developed to design targeted therapies against tumor spreading.

\section{Perspective}

The few areas of lymphatic research mentioned above and presented in the Reviews in this series are far from a complete list of 


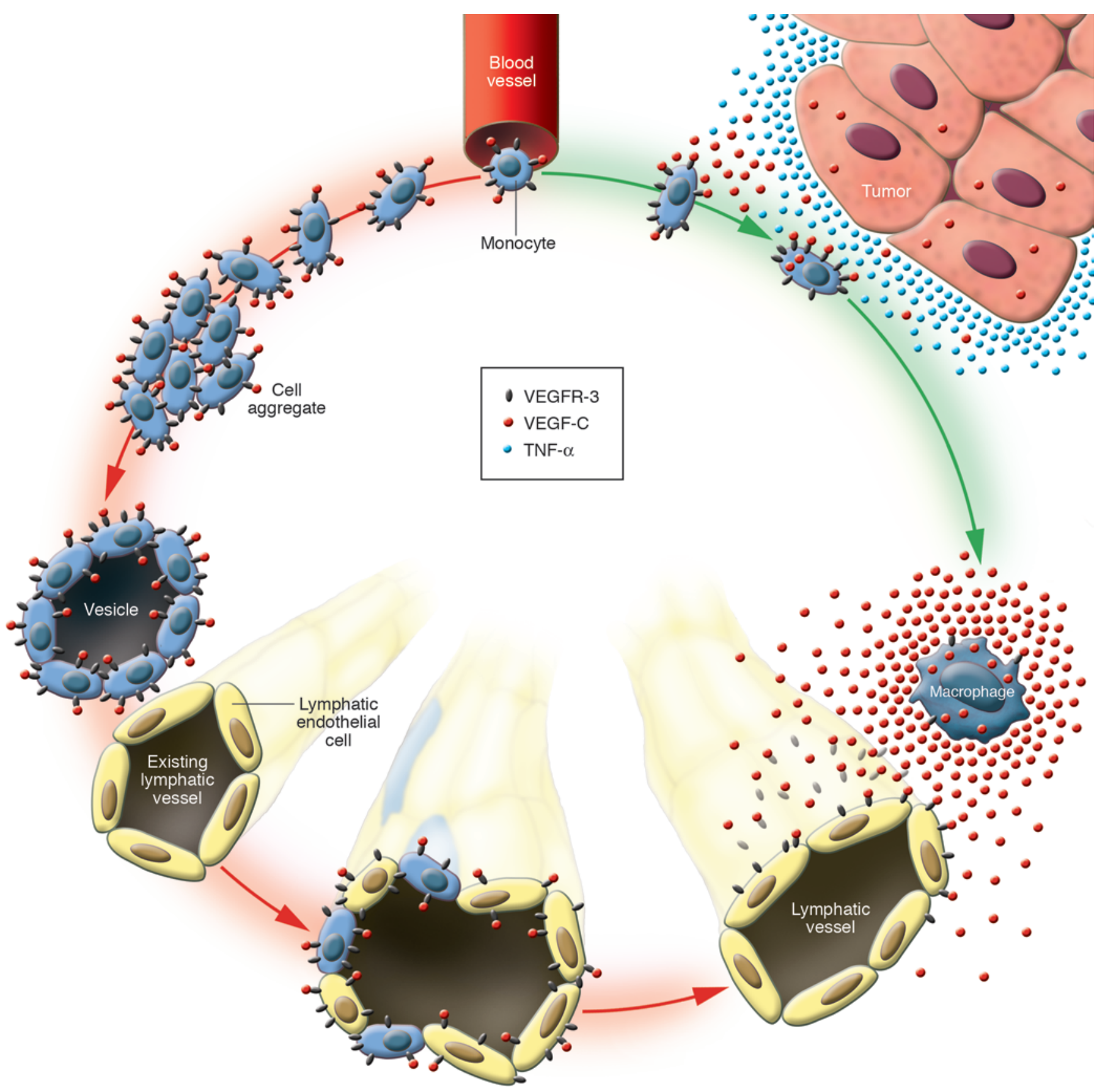

Figure 2

Contribution of $\mathrm{CD} 14^{+}$monocytes and macrophages to neolymphangiogenesis. VEGFR3 $/ \mathrm{CD} 14^{+}$circulating monocytes migrate from blood vessels and transdifferentiate into either LECs (left; red arrows) or, after appropriate stimulation, for example by tumor cells, into VEGFC-producing macrophages that induce growth of existing lymphatic vessels (right; green arrows). Reproduced from the Journal of Clinical Investigation (14).

fundamental biological aspects and associations with human diseases. Practical medical applications are still to be expected and may derive from an improved understanding of (a) the relation of lymphatics with adipocytes, (b) the mechanisms of inflammatory cell attraction and migration in lymphatics, and (c) the attraction and transport of infectious agents, ranging from bacteria to helminthes. In regenerative medicine, new concepts of treatment are required for nonsurgical therapy of lymphangiomas and secondary lymphedemas caused by surgical interruption of the lymphatic vasculature. Induction of lymphangiogenesis by transplantation of conditioned lymph nodes to surgically disrupted areas has already reached the clinic (36).

In more general terms, lymphatics are the first barrier for antigen-presenting cells that are transported into the next lymph node to initiate an adaptive immune response (31). Facilitating their entry could potentially improve the efficiency of vaccination. Since lymphatics also serve as conduits for tumor cells in metastatic spreading (35), specific interference could reduce or prevent 
tumor cell entry and implantation into the lymph node sinus. To mention yet another field, the significance of the lymphatic vascular system in diabetes has also just begun to emerge (37). Finally, there are several diseases that are caused by genetic or neoplastic alterations of the LECs, including genetic forms of lymphedema, as presented in the Review by Miikka Vikkula (38).

These examples for potential clinical applications of insights into lymph vessel biology and pathology are the beginning of an even longer list of applicable possibilities to modify diseases that will keep investigators engaged for years to come. The purpose of this Review series is not only to summarize the rapid development of the field of lymphatic vessel biology over the last 15 years and to emphasize its relevance to human disease, but also to impress on the reader how much uncharted scientific territory remains to be explored in this still nascent field.

\section{Acknowledgments}

This work was supported in part by EC Marie Curie Actions-ITN-FP7 Project 238756 (Translational Science for Vascular Inflammation and Remodeling).

Address correspondence to: Dontscho Kerjaschki, Clinical Department of Pathology, Medical University of Vienna, Allgemeines Krankenhaus, Währinger Gürtel 18-20, 1090 Vienna, Austria. Phone: 43.1.40400.5176; Fax: 43.1.40400.5193; E-mail: dontscho. kerjaschki@meduniwien.ac.at.
1. Azzali G. Historical notes on the lymphatic vascular system. Acta Biomed Ateneo Parmense. 1990; 61(3-4):113-125.

2. Wang W, et al. Inhibition of myosin light chain phosphorylation decreases rat mesenteric lymphatic contractile activity. Am J Physiol Heart Circ Physiol. 2009;297(2):H726-H734.

3. Petrova TV, et al. Defective valves and abnormal mural cell recruitment underlie lymphatic vascular failure in lymphedema distichiasis. Nat Med. 2004; 10(9):974-981.

4. Sevick-Muraca EM, Kwon S, Rasmussen JC. Emerging lymphatic imaging technologies for mouse and man. J Clin Invest. 2014;124(3):905-914.

5. Fawcett DW, Heidger PM, Leak LV. Lymph vascular system of the interstitial tissue of the testis as revealed by electron microscopy. J Reprod Fertil. 1969; 19(1):109-119.

6. Lohela M, Bry M, Tammela T, Alitalo K. VEGFs and receptors involved in angiogenesis versus lymphangiogenesis. Curr Opin Cell Biol. 2009; 21(2):154-165

7. Tammela T, Alitalo K. Lymphangiogenesis: molecular mechanisms and future promise. Cell. 2010; 140(4):460-476.

8. Kriehuber E, et al. Isolation and characterization of dermal lymphatic and blood endothelial cells reveal stable and functionally specialized cell lineages. J Exp Med. 2001;194(6):797-808.

9. Mäkinen $T$, et al. Isolated lymphatic endothelial cells transduce growth, survival and migratory signals via the VEGF-C/D receptor VEGFR-3. EMBOJ. 2001;20(17):4762-4773.

10. Zheng W, Aspelund A, Alitalo K. Lymphangiogenic factors, mechanisms, and applications. J Clin Invest. 2014;124(3):878-887.

11. Breiteneder-Geleff S, et al. Angiosarcomas express mixed endothelial phenotypes of blood and lymphatic capillaries: podoplanin as a specific marker for lymphatic endothelium. Am J Pathol. 1999; 154(2):385-394.

12. Wick N, et al. Lymphatic precollectors contain a novel, specialized subpopulation of podoplanin low, CCL27-expressing lymphatic endothelial cells. Am J Pathol. 2008;173(4):1202-1209.

13. Kang J, et al. An exquisite cross-control mecha- nism among endothelial cell fate regulators directs the plasticity and heterogeneity of lymphatic endothelial cells. Blood. 2010;116(1):140-150.

14. Kerjaschki D. The crucial role of macrophages in lymphangiogenesis. J Clin Invest. 2005; 115(9):2316-2319.

15. Schoppmann SF, et al. Tumor-associated macrophages express lymphatic endothelial growth factors and are related to peritumoral lymphangiogenesis. Am J Pathol. 2002;161(3):947-956.

16. Kerjaschki D, et al. Lymphatic endothelial progenitor cells contribute to de novo lymphangiogenesis in human renal transplants. Nat Med. 2006; 12(2):230-234.

17. Maruyama $\mathrm{K}$, et al. Inflammation-induced lymphangiogenesis in the cornea arises from CD11b-positive macrophages. J Clin Invest. 2005; 115(9):2363-2372.

18. Angeli V, et al. B cell-driven lymphangiogenesis in inflamed lymph nodes enhances dendritic cell mobilization. Immunity. 2006;24(2):203-215.

19. Kataru RP, et al. T lymphocytes negatively regulate lymph node lymphatic vessel formation. Immunity. 2011;34(1):96-107.

20. Kim H, Kataru RP, Koh GY. Inflammation-associated lymphangiogenesis: a double-edged sword? J Clin Invest. 2014;124(3):936-942.

21. Yang Y, Oliver G. Development of the mammalian lymphatic vasculature. J Clin Invest. 2014; 124(3):888-897.

22. Mortimer PS, Rockson SG. New developments in clinical aspects of lymphatic disease. J Clin Invest. 2014;124(3):915-921.

23. Wiig H, et al. Immune cells control skin lymphatic electrolyte homeostasis and blood pressure. J Clin Invest. 2013;123(7):2803-2815.

24. Machnik A, et al. Macrophages regulate salt-dependent volume and blood pressure by a vascular endothelial growth factor-C-dependent buffering mechanism. Nat Med. 2009;15(5):545-552.

25. Kopp C, et al. 23Na magnetic resonance imaging-determined tissue sodium in healthy subjects and hypertensive patients. Hypertension. 2013; 61(3):635-640.

26. Randolph GJ, Miller NE. Lymphatic transport of high-density lipoproteins and chylomicrons. J Clin
Invest. 2014;124(3):929-935.

27. Kerjaschki D, et al. Lymphatic neoangiogenesis in human kidney transplants is associated with immunologically active lymphocytic infiltrates. J Am Soc Nephrol. 2004;15(3):603-612.

28. Nykänen AI, et al. Targeting lymphatic vessel activation and CCL21 production by vascular endothelial growth factor receptor-3 inhibition has novel immunomodulatory and antiarteriosclerotic effects in cardiac allografts. Circulation. 2010; 121(12):1413-1422.

29. Stuht S, et al. Lymphatic neoangiogenesis in human renal allografts: results from sequential protocol biopsies. Am J Transplant. 2007;7(2):377-384.

30. Ruddle NH. Lymphatic vessels and tertiary lymphoid organs. J Clin Invest. 2014;124(3):953-959.

31. Card CM, Yu SS, Swartz MA. Emerging roles of lymphatic endothelium in regulating adaptive immunity. J Clin Invest. 2014;124(3):943-952.

32. Swartz MA, et al. Tumor microenvironment complexity: emerging roles in cancer therapy. Cancer Res. 2012;72(10):2473-2480.

33. Kerjaschki D, et al. Lipoxygenase mediates invasion of intrametastatic lymphatic vessels and propagates lymph node metastasis of human mammary carcinoma xenografts in mouse. J Clin Invest. 2011; 121(5):2000-2012.

34. Liersch R, Hirakawa S, Berdel WE, Mesters RM, Detmar M. Induced lymphatic sinus hyperplasia in sentinel lymph nodes by VEGF-C as the earliest premetastatic indicator. Int J Oncol. 2012; 41(6):2073-2078.

35. Karaman S, Detmar M. Mechanisms of lymphatic metastasis. J Clin Invest. 2014;124(3):922-928.

36. Tammela T, et al. Therapeutic differentiation and maturation of lymphatic vessels after lymph node dissection and transplantation. Nat Med. 2007; 13(12):1458-1466.

37. Haemmerle M, et al. Enhanced lymph vessel density, remodeling, and inflammation are reflected by gene expression signatures in dermal lymphatic endothelial cells in type 2 diabetes. Diabetes. 2013; 62(7):2509-2529.

38. Brouillard P, Boon L, Vikkula M. Genetics of lymphatic anomalies. J Clin Invest. 2014; 124(3):898-904. 